\title{
La Previsión Episódica en la Etapa Pre-Escolar: Equivalencia entre Medidas y su Relación con los Procesos de Orientación Futura y la Teoría de la Mente
}

\author{
Episodic Foresight in Preschool Age: Equivalence between Measures \\ and the Relation with Future Oriented Processes and the Theory of Mind
}

\author{
Alejandro Vásquez Echeverría* \\ Universidad de la República, Montevideo, Uruguay
}

\begin{abstract}
Resumen
Este trabajo examina la equivalencia entre diferentes medidas de previsión episódica y su relación con los procesos orientados a futuro, y la teoría de la mente en la edad pre-escolar. Los procesos orientados a futuro y la previsión episódica (PE), han sido señalados como componentes importantes para ciertos logros evolutivos como la planificación, la auto-regulación y adaptación social. También se ha postulado que pensamiento futuro episódico y teoría de la mente están relacionados, en virtud de su sustrato neural compartido. El primer estudio tuvo por objetivo analizar la coherencia de desarrollo entre las medidas de PE, y de estas con la postergación de la gratificación auto-impuesta controlando según la aptitud general de razonamiento, y participaron 15 niños de 3 y 4 años. El segundo, tuvo por objetivo explorar la relación entre PE, los procesos orientados a futuro, la teoría de la mente controlando según lenguaje, participando 78 niños de 4 años. Los resultados muestran que las diferentes medidas para evaluar la PE son equivalentes. También se encontró un alto grado de coherencia comportamental entre las medidas evaluadas. Sin embargo, los datos no aportan evidencia evolutiva que permitan incluir a la PE dentro de los constructos más amplios estudiados. Palabras clave: Previsión episódica, procesos de orientación futura, Teoría de la Mente, cognición episódica, prospección.
\end{abstract}

\begin{abstract}
This paper explores the equivalence between episodic foresight measures and its relationship with future oriented processes and the theory of mind in preschoolers. Future oriented processes and episodic foresight have been indicated as a grounding basis of important developmental achievements such as planning, self-regulation and social adaptation. Also, it was postulated that the theory of mind and episodic foresight are related due to a shared common neural substrate. The objective of the first study, in which 15 children aged 3 and 4 participated, was to explore the developmental coherence between episodic foresight measures and the self-imposed delay of gratification controlled by the reasoning ability. In Study 2, 78 children were tested in episodic foresight, future oriented processes and theory of mind controlled by the language ability. Results show that the different measures of episodic foresight are highly equivalent. Also, in both studies a high behavioral coherence between all the measures analyzed was found. Nevertheless, data do not support the inclusion of episodic foresight in the broader constructs studied.

Keywords: Episodic foresight, future oriented processes, Theory of Mind, episodic cognition, prospection.
\end{abstract}

La capacidad para pensar en el futuro, esencial para procesos como tomar decisiones, planificar y lograr objetivos, es considerada una ventaja adaptativa en la evolución

* Dirección de envio: Instituto de Fundamentos y Métodos, Facultad de Psicología, Universidad de la República, Tristan Narvaja, 1674, Montevideo, Uruguay 11200. E-mail: avasquez@psico.edu.uy

Agradezco a la Dra. Orlanda Cruz sus comentarios y valiosas sugerencias realizadas sobre un borrador de este manuscrito. Lucía Álvarez también colaboró con la lectura y corrección atenta de una de las versiones finales del mismo. humana (Suddendorf \& Corbalis, 2007). Dentro de la psicología del desarrollo, recientemente ha surgido un área de estudio sobre la capacidad para simular mentalmente eventos futuros personales y consecuencias de las acciones. Esta competencia emerge entre los 3 y 5 años, empero, aun son escasos los datos sobre en qué medida la PE, los procesos orientados a futuro y la capacidad de representar el punto de vista de los otros (teoría de la mente) se asocian entre sí en la etapa pre-escolar.

Este comportamiento orientado hacia el futuro episódico ha sido estudiado o conceptualizado de diversas 
formas, entre ellas prospección, descentramiento temporal, viaje mental en el tiempo o pensamiento futuro episódico (Atance \& O'Neill, 2001; Buckner \& Carroll, 2007; McCormack \& Hoerl, 2008). Recientemente, Suddendorf y Moore (2011) propusieron unificar todos estos conceptos bajo el nombre de previsión episódica (en adelante, PE). Este término será el preferido en este trabajo en tanto facilita la construcción taxonómica por basarse en la distinción propuesta por Tulving (1984) entre memoria episódica y memoria semántica. Atance y O'Neill (2001) fueron quienes propusieron que la misma distinción aplicada a la memoria puede aplicarse para el pensamiento futuro, distinguiendo el pensamiento futuro semántico del episódico. El pensamiento futuro semántico se trataría de un pensamiento noético, saber sobre el funcionamiento del mundo físico o social basados en la rutina o en procesos con alta probabilidad de repetirse (e.g. en psicología cognitiva los scripts). Por ejemplo, ser capaz de pensar lo que sucederá en un restaurante (ejemplo social) o imaginar la trayectoria de una piedra a lanzar sobre un barranco o despeñadero (ejemplo físico), parece emerger previamente en el desarrollo ontogenético en tanto el conocimiento de guiones parece consolidarse sobre los 3 años (Hudson, Shapiro, \& Sosa, 1995) y el conocimiento de probables trayectorias de objetos físicos puede emerger incluso antes, en los primeros dos años de vida (Teglás, Girotto, Gonzalez, \& Bonatti, 2007). En cambio, la PE fue definida por Atance y O'Neill (2001) como " an ability to project the selfforward in time to pre-experience an event" (p. 537). Así, la PE refiere a la capacidad para construir mentalmente representaciones sobre eventos futuros con tres características: (a) que sea una anticipación de deseos, necesidades o expectativas que proyecta al self hacía un momento futuro, de forma sensorial, emocional y/o cognitiva; (b) que esa anticipación pueda desligarse del estado motivacional actual de la persona y; (c) que pueda ser ubicada en un momento y lugar aproximado.

\section{Relaciones entre la Previsión Episódica con Otros Procesos Cognitivos}

Se ha relacionado a la PE con otros procesos y funciones cognitivas, al menos en dos sentidos: (a) aquellos procesos que necesariamente requieren utilización de recursos mentales provistos por la $\mathrm{PE}$, como los procesos orientados a futuro; $\mathrm{y}(\mathrm{b})$ otras funciones, incluidas dentro de un concepto más amplio, como por ejemplo el de autoproyección (Buckner \& Carroll, 2007).

\section{La Previsión Episódica como un Proceso Orientado a Futuro}

Los procesos de orientación a futuro son definidos como los procesos que permiten actuar en el presente para obtener resultados distantes de la conducta. Tradicionalmente en ellos se incluye la planificación, la postergación de la gratificación y la memoria prospectiva (Heith, 1997) y se ha sugerido que la PE podría ser uno más de estos procesos (Szpunar, 2010). La capacidad de planificación, refiere a la posibilidad de incluir eventualidades y coordinar conductas en el presente para alcanzar estados futuros. Requiere de pensamiento representacional, control sobre la acción y también, de la anticipación de futuros estados del self (Heith, 1997). Puede distinguirse entre planificación donde el self está implicado (que requiere PE) de planificación operativa, donde no está necesariamente implicado un futuro estado del yo. La postergación de la gratificación, definida como la habilidad para posponer la gratificación inmediata y persistir en la búsqueda de una meta que conduce a la obtención de resultados posteriores, también requiere de $\mathrm{PE}$, en tanto el mismo permite imaginar los beneficios futuros que posibilitan la toma de decisión por medio de la expectación e imaginación por el estado futuro, o la utilidad económica puesta en el futuro (Bembenutty \& Karabenick, 2004). Por su parte, la memoria prospectiva, definida como la habilidad para recordar ejecutar actividades pretendidas en el futuro y activar dicho recuerdo en el momento correspondiente, también requeriría de $\mathrm{PE}$, en tanto la codificación se realiza estableciendo una intención en la que el self ejecutará algo en el futuro, que luego debe mantenerse en el tiempo y, por último, cambiar de tarea cuando el estímulo se presenta (Kliegel, Martin, McDaniel, \& Einstein, 2002). Tanto la postergación de la gratificación, la planificación y la memoria prospectiva son capacidades que se incrementan sensiblemente en los años pre-escolares. La relación empírica entre estos fenómenos está aun débilmente explorada, especialmente en el periodo pre-escolar. Atance y Jackson (2009) han mostrado que estos procesos pueden estar sujetos a un desarrollo conjunto, aunque sus resultados no permitieron apoyar la idea de unidad de constructo entre los procesos de orientación futura con la PE ya que las correlaciones se reducen sensiblemente cuando se controla por edad y lenguaje receptivo.

\section{La Previsión Episódica como Parte de la Auto-Proyección}

El concepto de autoproyección, propuesto por Buckner y Carroll (2007) postula que la PE, la teoría de la mente, la memoria prospectiva y la navegación son parte del mismo constructo, por lo que los autores propugnan por estudiarlas conjuntamente. Esta idea está en contra de las nociones modulares para el estudio de la teoría de la mente (Scholl \& Leslie, 1999), pero con evidencia anatómica y funcional de los estudios de neuroimagen, Buckner y Carroll mostraron que la red neuronal requerida para la proyección hacia otro tiempo (pasado o futuro) o perspectiva (de otra persona o de otro punto de vista) es común a estos procesos y diferenciada de otras tareas que requieren resolución de problemas o simple imaginación. Estos procesos estarían ligados entre sí en tanto las experiencias pasadas serían utilizadas de forma adaptativa para imaginar eventos y otras perspectivas, no derivadas del ambiente inmediato. Desde el punto de vista conceptual, la asociación entre PE y teoría de la mente tiene mucho sentido. La PE, en especial su componente de imaginar nuestros propios estados 
Echeverría, A. V. (2015). La Previsión Episódica en la Etapa Pre-Escolar: Equivalencia entre Medidas y su Relación con los Procesos de Orientación Futura y la Teoría de la Mente.

mentales futuros, está relacionado a reconocer que otros tienen -al mismo tiempo que nosotros- un estado mental diferente. Ambas habilidades aparecen interrelacionadas en los casos dónde se prevé, desde las consecuencias de nuestras acciones, estados mentales futuros de otras personas. Sin embargo, Rosenbaum, Stuss, Levine y Tulving (2007) ponen en causa la hipótesis de la auto-proyección en la población adulta, en tanto pacientes amnésicos que carecían de conciencia autonoética (i.e., de la consciencia del tiempo personal), eran capaces de representar adecuadamente los estados mentales de otros sujetos. Esto sugiere la modularidad de estas funciones, o sea, que se tratarían de módulos de procesamiento de la información con especialización funcional y localización específica, dónde la presencia de uno no permite compensar la ausencia del otro. En cuanto a la etapa pre-escolar, datos empíricos de naturaleza comportamental mostraron que la teoría de la mente está relacionada con algunos procesos orientado a futuro en niños de 3 y 5 años (Bischof, 2008) - sin aportar datos específicos que analicen la relación específica con la PE. Por otro lado, la teoría de la mente ha sido asociada con el desarrollo del funcionamiento ejecutivo en su conjunto o alguno de sus componentes, tendencia que permaneció aun cuando se controló según la inteligencia o la edad en meses (Carlson \& Moses 2001; Carlson, Moses, \& Claxton, 2004). Sin embargo, aún se carecen de datos sobre la trayectoria evolutiva y coherencia comportamental entre la teoría de la mente con la PE en la etapa de emergencia de la capacidad. Si bien se ha sugerido que ambos procesos emergen entre los 3 y los 5 años, no hay datos sobre la variación intra-individual.

\section{Equivalencia entre los Experimentos Propuestos para Evaluar la PE}

Debido a la dificultad de evaluar la fiabilidad de las respuestas sobre el futuro personal, el aspecto metodológico siempre ha sido una fuente de debate y se ha planteado la necesidad de ejecutar nuevos experimentos para evaluar la PE. En la literatura sobre la PE se utilizan muchos experimentos para evaluar el futuro episódico preguntando al niño directamente sobre necesidades, deseos o eventos futuros, pero sin un desplazamiento temporal. Un ejemplo tradicional para evaluar la $\mathrm{PE}$ es preguntar al niño ¿qué vas a hacer mañana? Si la respuesta es probable de acontecer, según la evaluación de los padres, se otorga un punto. Sin embargo, Russell, Alexis y Clayton (2010) plantearon que estos experimentos plantean problemas para inferir la presencia de PE, y sugieren que los métodos de test de cuchara son más convenientes. En los experimentos diseñados bajo este paradigma, los niños deben seleccionar ítems para un uso futuro, y la corrección de estas respuestas sólo es analizada posteriormente, cuando ese uso futuro se consuma. Estos experimentos además pueden incluir un desplazamiento espacial real (hacia otro lugar). No obstante, no hay estudios hasta la fecha que hayan explorado la posible equivalencia en el rendimiento de los niños pre-escolares entre los diversos formatos de las tareas diseñadas para evaluar la $\mathrm{PE}$, bajo paradigmas metodológicos de "test de cuchara" o sin un desplazamiento temporal o espacial efectivo.

En este contexto, este trabajo se propone los siguientes objetivos. Primero, determinar la equivalencia entre diversos tipos de experimentos para evaluar la PE. Segundo, determinar la coherencia evolutiva en la emergencia de estas competencias en la etapa pre-escolar. Tercero y vinculado al objetivo anterior, determinar si los modelos de conceptualización de la PE en constructos más amplios (i.e.: los procesos de orientación futura y auto-proyección) reúnen evidencia empírica en la etapa pre-escolar. Para esto se proponen dos estudios. En el primero se explora la validez y equivalencia de diferentes tipos de experimentos para evaluar la PE y de forma exploratoria, la asociación con un proceso de orientación futura, controlando según la aptitud general de razonamiento. En el segundo estudio, se explora en mayor profundidad la relación de la PE con los procesos de orientación futura y con un constructo central de la teoría de la auto-proyección, la teoría de la mente, controlando según el desarrollo lingüístico.

\section{Estudio 1}

\section{Objetivo}

Este primer estudio tuvo por objetivo explorar el grado de equivalencia de medida entre experimentos de PE con desplazamiento temporo/espacial con dos sin desplazamiento espacial. Asimismo, se pretende explorar la coherencia en el desarrollo comportamental de la PE con un proceso de orientación a futuro (la postergación de la gratificación auto-impuesta) controlando según la aptitud general de razonamiento en los pre-escolares.

\section{Método}

\section{Participantes}

Participaron 15 preescolares (9 niñas) de 3 y 4 años $(M=44.27$ meses, $S D=3.71)$ provenientes de familias de clase media de la región de Guipúzcoa (España), que tenían como lengua materna el castellano. Se trató de una muestra de conveniencia, reclutada a través de avisos en centros pre-escolares o bola de nieve. Cuatro evaluaciones adicionales no fueron completadas por inhibición (3 casos) o desatención/desinterés (1 caso) de los niños. Se obtuvo consentimiento informado escrito de los padres previo a la sesión experimental.

\section{Instrumentos \\ Previsión Episódica}

Tarea del Viaje. En esta tarea se le mostraron al niño 8 objetos reales de los cuales debía seleccionar tres para irse de viaje con papá o mamá. Se le pidió al niño que tomase 3 objetos, los coloque en una mochila y que explicase por qué los seleccionó. En base a las respuestas verbales se realizó una codificación sobre la cual se atribuyó la presencia o no de PE. La prueba y los procedimientos de 
codificación se describen con detalle en Atance y O'Neill (2005). Se codificó positivamente las respuestas que incluían expresiones del castellano con valor de futuro (Ródenas, Brito, \& Carranza, 1991; e.g.: si + pretérito imperfecto del indicativo; formas de condicional; voy/ vamos $a+$ infinitivo), en primera persona, y que incluyeran un posible estado futuro del self (e.g.: hambre, sed o frío) que pudieran ser satisfechos con el objeto seleccionado (Rango: 0-3 puntos).

Tarea de Dibujo. Esta tarea consiste en solicitar a los niños que planifiquen lo que van a dibujar (Atance \& O’Neill, 2005). La tarea tiene como constricción que se tienen que utilizar las hojas previamente diseñadas y utilizar la figura que allí aparece como parte del dibujo (una línea y un círculo). Se otorga un punto por cada dibujo correctamente realizado en función de la intención expuesta inicialmente, en tanto para realizar correctamente la tarea el niño tiene que anticipar lo que el mismo es capaz de hacer en el futuro (Rango: 0-2).

Tarea de los Cuartos. Esta tarea desarrollada por Suddendorf y Busby (2005) pretende evaluar la capacidad de los preescolares de evitar un estado futuro de aburrimiento personal. Aquí se describe la versión adaptada al contexto español. Se llevaba al niño a una primera sala, donde solo había hojas y cuadernos para colorear y pintar. Luego de un breve lapso en la Sala A (habitación de la Pantera Rosa), dónde el experimentador enfatizaba la presencia del material gráfico (poster de la Pantera Rosa) que caracterizaba la sala y la sola presencia de hojas y cuadernillos para dibujar y pintar, se llevaba al niño a una segunda sala, la Sala B, donde había diversos objetos y se jugaba por 5 minutos a juegos no relacionados. Luego se mostraban al niño 4 objetos colocados por el experimentador con los que el niño podía interactuar (objeto diana: lápices de colores, distractores: una corneta, ropa de Mickey -sin el peluche-, piezas aisladas de puzle). Luego, se le decía al niño: "¿te acuerdas de la sala de la Pantera Rosa? ¿Ahora vamos para allí a estar en la sala por un tiempo, cuál de estas cosas quieres llevar contigo?" En ese momento se le pedía al niño que escogiera uno de los cuatro objetos que tenía a su frente, estando entre ellos los lápices para dibujar y colorear. En este estudio, la selección del objeto faltante (materiales para dibujar y colorear) y su utilización en el cuarto de la Pantera Rosa implicó la asignación de un punto (Rango: 0-1).

\section{Postergación de la Gratificación Auto-Impuesta}

Premio Diferido. Se utilizó el paradigma de Mischel, Shoda y Peake (1988). Se le transmite al niño que por su participación en la tarea le correspondía un chocolate o una golosina. Inmediatamente, el niño era informado que el experimentador debía hablar con la maestra y consignaba lo siguiente: "mientras tanto, tu esperas aquí. Si no comes el chocolate/golosina hasta que yo vuelva te voy a dar 3 más. Si en algún momento quieres comenzar a comerlo, puedes tocar este timbre que yo vuelvo enseguida". El experimentador se retiraba de la sala y volvía a los 4 minu- tos, salvo que el niño activara el timbre inalámbrico. La puntuación fue calculada en base a los minutos de espera (Rango: 0-4).

\section{Habilidad General de Razonamiento}

Escala de Madurez Mental Columbia. Se evaluó la aptitud general de razonamiento mediante la Escala de Madurez Mental Columbia (Burgemeister, Hollander, \& Lorge, 1983), por la cual el niño debe formular leyes o reglas para determinar la inclusión en clases. En cada lámina que se le presenta, hay tres, cuatro o cinco objetos y siempre uno de ellos se encuentra excluido de la clase o las dos clases que existen (i.e.: en las láminas con 5 objetos puede haber dos clases). El niño debe señalar cuál es el objeto que no podía ser incluido junto con otro en una misma clase. Se utilizó el Índice de Madurez Mental (IMM) de dicha escala para los análisis estadísticos.

\section{Procedimiento}

El niño era recibido en un salón apartado de dos centros educativos que consintieron colaborar en el proyecto o en un contexto de laboratorio. Luego de una breve presentación personal, se le consignaba al niño que se iban a realizar una serie de tareas, junto a la compañía del peluche Pocoyó. La sesión duró aproximadamente media hora y era grabada para posterior análisis del material textual. El orden de las pruebas fue contrabalanceado. El índice de concordancia kappa, fue calculado por dos jueces entrenados con casos ficticios. Los índices, para la totalidad de la muestra, fueron de $k=.76$ para la tarea del viaje y $k=$ .78 para la tarea de dibujo, ambos considerados buenos (Landis \& Koch, 1977).

\section{Resultados y Discusión}

El análisis de varianza ANOVA mostró que el rendimiento de los niños no varió significativamente en función del sexo, forma de presentación de las tareas, o lugar de evaluación por lo que los datos fueron tratados unitariamente. Igualmente debe señalarse que la tarea de premio diferido presenta una diferencia tendiente a la significación a favor de las niñas, $F(1,13)=4.49, p=.05, d$ de Cohen $=$ 1.03. Se realizó un análisis de correlación entre las tareas experimentales con la edad en meses y el IMM. En la Tabla 1 puede apreciarse los estadísticos descriptivos así como los valores de las correlaciones, que son mayormente altos y estadísticamente significativos. La tarea de premio diferido mantiene correlaciones significativas con la tarea de viaje, la de dibujo y el IMM.

A pesar de que las correlaciones de orden cero representan un dato valioso, es importante determinar qué sucede cuando son controladas según la edad en meses y el IMM. Si las mismas se mantienen en valores similares, se puede asumir que se está frente a constructo conceptual no influenciado por dichas variables. Caso contrario, si los valores se reducen sustancialmente, esto puede atribuirse a los efectos del desarrollo simultáneo 
Echeverría, A. V. (2015). La Previsión Episódica en la Etapa Pre-Escolar: Equivalencia entre Medidas y su Relación con los Procesos de Orientación Futura y la Teoría de la Mente.

Tabla 1

Medias, Desviaciones Típicas (SD) y Correlaciones entre las Tareas Experimentales e Índice de Madurez Mental

\begin{tabular}{|c|c|c|c|c|c|}
\hline & $M(S D)$ & 2 & 3 & 4 & 5 \\
\hline 1.Tarea de Viaje & $1.01(1)$ & $.67^{* *}\left[.75^{* *}\right]$ & $.47 *[.49 *]$ & $.71 * *[.67 * *]$ & .36 \\
\hline 2. Tarea de Dibujo & $.87(.64)$ & - & $.62 * *[.60 * *]$ & $.39 *[.58 * *]$ & .10 \\
\hline 3. Tarea de los Cuartos & $.27(.45)$ & & - & $.17[.17]$ & .17 \\
\hline 4. Premio Diferido & $2.8(1.6)$ & & & - & $.57 * *$ \\
\hline 5. IMM & 84 (13.6) & & & & - \\
\hline
\end{tabular}

Nota. Entre paréntesis rectos [ ] se ofrecen los valores de la correlación parcial controlado por edad en meses y madurez mental. IMM = Índice de Madurez Mental.

$* p<.05 ; * * p<.01$.

de las competencias requeridas para resolver las tareas o a los requerimientos cognitivos implicados por la tarea. La correlación parcial de segundo orden (controlando por edad en meses e IMM) muestra que las mismas se mantienen en valores altos. Las correlaciones parciales muestran un patrón similar al visto en las correlaciones de orden cero, a excepción de la correlación entre la tarea de premio diferido y la tarea de los cuartos, que presenta un valor bajo y no significativo.

Los resultados muestran que las capacidades de los niños para resolver las diferentes tareas que exigen PE son coherentes. Esto sugiere que este constructo está siendo evaluado adecuadamente mediante las tareas que se han propuesto en la literatura, más allá de que presenten ciertas diferencias. Estas diferencias remiten a la diferente naturaleza fenomenológica de los aspectos de la PE, por ejemplo, la tarea de dibujo no implica una anticipación de necesidades sino de las capacidades del self para realizar una tarea; en la tarea de viaje la proyección para la anticipación de necesidades es imaginada y no real; en la tareas de los cuartos hay desplazamiento espacial. Sin embargo, las mismas parecen requerir de una capacidad cognitiva común para ser completadas. Por otro lado, el hecho de que los valores de las correlaciones parciales y su significación estadística se mantengan altos, puede interpretarse en línea con la posible modularidad del constructo. Esto es, el desarrollo de la PE sigue un curso no directamente relacionado a la variable remota edad o a la madurez mental del niño. Los datos de las correlaciones parciales entre las tareas de PE y premio diferido alcanzan también niveles significativos (a excepción de la correlación entre premio diferido y tarea de los cuartos), pero que no son tan elevados como las tareas de PE entre sí. Esto puede deberse a que si bien la postergación de la gratificación requiere en gran parte de la PE, también demanda de otros comportamientos de auto-regulación (e.g.: inhibición). Este requerimiento de componentes múltiples para postergar la gratificación puede implicar que, si bien un niño puede imaginarse en el futuro-obteniendo un beneficio mayor-, igualmente no pueda tolerar la espera y actúe.

Vistos estos datos del Estudio 1, queda por responder si (a) la PE es un constructo independiente o muestra coherencia evolutiva con otros procesos orientados a futuro o la teoría de la mente y; (b) que sucede si la asociación entre estos constructos es controlada según el lenguaje (ya que en el primer estudio se utilizó una medida que refleja las habilidades generales de razonamiento). Para profundizar en estos puntos se realizó el Estudio 2.

\section{Estudio 2}

\section{Objetivo}

El objetivo de este estudio fue explorar la coherencia comportamental en el desarrollo de la PE con los procesos orientados a futuro, y un componente de la auto-proyección (la teoría de la mente) controlando según el lenguaje receptivo para determinar su posible pertenencia a un mismo constructo.

\section{Método}

\section{Participantes}

Esta vez, fue incluida una muestra mayor y diversa desde el del punto de vista socio-económico, para mejorar la posibilidad de generalización de los datos. Participaron 78 niños (39 niñas) de 4 años $(M=54.4 ; S D=3.51)$ de desarrollo normal, de contextos socio-económicos mediosaltos, medios y bajos del área metropolitana de Montevideo (Uruguay). Ocho centros educativos de barrios con poblaciones de diversas características socio-económicas fueron contactados y aceptaron colaborar con el estudio. Se obtuvo consentimiento informado por parte de los padres previo al trabajo experimental. Otras 8 evaluaciones no fueron terminadas por desatención o desinterés de los niños (siete casos) o error experimental (un caso). 


\section{Instrumentos}

\section{Pensamiento Futuro Episódico}

Tarea del Libro de Viaje. Evalúa en qué medida el niño es capaz de anticipar estados futuros de necesidad o deseo propios. Si bien es una tarea muy similar a la tarea del viaje del Estudio 1, fue seleccionada esta variación por dos razones (a) para garantizar un mayor rango de puntuación y (b) evitar sesgos por la saliencia de algunos objetos (por color o tamaño). Se utilizó la versión de Atance y Meltzoff (2005) por la cual se consigna que el niño debe ayudar a su papá o mama a preparar un viaje. Posteriormente se le presentan fotos de lugares donde se irá de viaje y se identifica el lugar junto con el experimentador. Seguidamente se presentaban fotos de 3 objetos, de los cuales uno solo era relevante para el lugar del viaje y los otros funcionaban como distractores. Se le preguntaba al niño “¿Cuál de estos llevarías contigo al lugar x (foto del destino)?", y luego de elegir el ítem, se preguntaba: “¿Por qué llevarías x (objeto seleccionado)?”. Los destinos y los objetos presentados a elección (presentados entre paréntesis) fueron los siguientes: (a) un desierto (objeto diana: una botella de agua; distractores: un bol y un cd); (b) una calle de ciudad bajo lluvia (objeto diana: chaqueta; distractores: un regalo y una radio) y; (c) una montaña con rocas (objeto diana: tirita; distractores: un libro y piedras). El orden de presentación fue contrabalanceado. Se otorgaba un punto por cada objeto diana seleccionado $\mathrm{y}$ un punto por una respuesta verbal con valor de futuro y que presente un estado futuro del self, como fue descrito para el Estudio 1 (Rango: 0-6).

Tarea de Dibujo. El mismo procedimiento al trabajo descrito en el Estudio 1.

\section{Teoría de la Mente}

Tarea de Contenido Inesperado. Evalúa un aspecto de la teoría de la mente que refiere a las expectativas de contenido que tendría el extraño, luego de haber sido engañado uno mismo (Gopnik \& Astington, 1988). Se presentó al niño una caja de dentífrico y se le preguntaba al niño "que piensas que tiene la caja dentro?". Una vez obtenida la respuesta dentífrico (o un cepillo dental), se abría la caja. El niño, para su sorpresa se encontraba con lápices. Luego de esta comprobación, se preguntó: (a) “qqué pensabas que habia dentro de la caja antes de abrirla?" y (b) "si ahora entrara tu madre/padre a la sala, ¿qué pensaría que hay dentro de la caja?". Un punto obtenía cada respuesta correcta siempre y cuando se contestara correctamente la pregunta control: ¿Qué hay dentro de la caja realmente? (Rango: 0-2).

\section{Planificación}

Torre de Hanoi. Se trata de una tarea destinada a evaluar la capacidad de planificación. Se utilizó la versión propuesta por Carlson et al. (2004): a los niños/as se les presentó una estructura de madera con tres estacas y dos discos. A los efectos de comprensión, se consignó que el juego se llama El país de los monos dónde las estacas representan árboles, el disco grande el Papá mono y el disco pequeño es el/la Niño/a mono/a. A partir del nivel tres se incorpora el Bebé mono (un disco más pequeño). Se le explicó al participante que el juego tiene 3 reglas: (a) que los discos (monos) deben permanecer en las estacas (árboles); (b) que solo un mono puede saltar a la vez y; (c) que un mono más grande nunca puede sentarse sobre un mono más chico - ningún disco más grande puede ser colocado sobre un disco más pequeño en la misma estaca. Así el papá mono nunca puede ser colocado por encima del nene mono, ni del bebe mono. Se solicitó al niño que ejemplificase con alguna acción que las ha comprendido. El experimentador contaba con un set propio dónde mostraba la posición final que se debía alcanzar. El niño disponía de dos chances por nivel y cada error era corregido y explicado solo en el primer ensayo (Rango: 0-6).

\section{Postergación de la Gratificación}

Premio Diferido. El mismo procedimiento al descrito en el Estudio 1, salvo que para aumentar la variabilidad entre los niños de 4 años, la espera fue de 8 minutos (Rango: 0-8).

\section{Memoria Prospectiva}

Recuperación del Animal. Evalúa en qué medida la persona es capaz de recordar algo en el futuro. Se le mostraba al niño un animal de la selva y se le preguntaba si lo quería llevar de regalo. Ante la respuesta afirmativa (todos los casos), se procedía a explicarle que se lo llevaría si recordaba pedírselo al experimentador al terminar otra de las tareas (PPVT III; Rango: 0-1).

\section{Lenguaje Receptivo}

Test de Vocabulario en Imágenes Peabody (PPVT III). Se utilizó la versión en castellano (Dunn, Dunn, \& Arribas, 2006). Evalúa el nivel de vocabulario receptivo y la aptitud verbal.

\section{Procedimiento}

Los participantes fueron recibidos en una sala apartada del centro educativo. Luego de una pequeña conversación, se explicó al niño que se jugaría junto al peluche de Barney a diferentes tareas. Siguiendo la lógica de Carlson y Moses (2001) se aplicaron los experimentos siguiendo este orden: tarea de dibujo, tarea de viaje, tarea de contenido inesperado, torre de Hanoi, postergación de la gratificación y memoria prospectiva. La codificación fue realizada por dos jueces independientes, entrenados con el $15 \%$ de los casos, que utilizaron los mismos criterios a los presentados en el Estudio 1 para la tarea de viaje y de dibujo. El índice de concordancia kappa fue $k$ $=.94$ para la tarea de viaje y de $k=.93$ para la tarea de dibujo (en base al 78.8\% de la muestra), los cuales son altos (Landis \& Koch, 1977). 
Echeverría, A. V. (2015). La Previsión Episódica en la Etapa Pre-Escolar: Equivalencia entre Medidas y su Relación con los Procesos de Orientación Futura y la Teoría de la Mente.

\section{Resultados y Discusión}

En la Tabla 2 se pueden consultar las medias y las correlaciones entre las diversas tareas experimentales y el PPVT-III. En tanto no se encontraron diferencias según sexo, los resultados fueron tratados de forma integrada. Las diferencias entre niños y niñas en la tarea de premio diferido fueron tendientes a la significación, $F(1,76)=3.87 ; p$ $=.05 ; d$ de Cohen $=.43$. La mayoría de las correlaciones de orden cero arrojan valores moderados y altos, además de significativos. Como se puede apreciar en la Tabla 2, un resultado relevante es la alta coehrencia comportamental entre las diversas tareas que evalúan la $\mathrm{PE}$ y los procesos de orientación futura, similares a los reportados en el Estudio 1 y por Atance y Jackson (2009). Aquí se añade la comprobación de que las pruebas destinadas de PE también correlacionan significativamente con las puntuaciones en teoría de la mente.

Tabla 2

Media, Desviaciones Típicas(SD) y Correlaciones entre las Tareas Experimentales y Lenguaje Receptivo

\begin{tabular}{|c|c|c|c|c|c|c|c|}
\hline & $\mathrm{M}(S D)$ & 2 & 3 & 4 & 5 & 6 & 7 \\
\hline 1.Tarea de Viaje & $3.24(2)$ & $.45 * *[.28 * *]$ & $.34 * *[.14]$ & $.36 * *[.08]$ & $.23 *[-.02]$ & $.20 \dagger[-.04]$ & $.60 * *$ \\
\hline 2.Tarea de Dibujo & $.94(.78)$ & - & $.32 * *[.18 \dagger]$ & $.36^{* *}[.19 \dagger]$ & $.10[-.06]$ & $.24 *[.10]$ & $.42 * *$ \\
\hline 3.Premio Diferido & $4.37(3.5)$ & & - & $.47^{* *}\left[.34^{* *}\right]$ & $.20 \dagger[.07]$ & $.31 * *[.19 \dagger]$ & $.40 * *$ \\
\hline 4.Torre de Hanói & $2.7(1.7)$ & & & - & $.22 \dagger[.05]$ & $.41 * *[.28 * *]$ & $.50 * *$ \\
\hline 5.Recuperación del Animal & $.36(.48)$ & & & & - & $.23 *[.10]$ & $.36^{* *}$ \\
\hline 6.Contenido Inesperado & $1.04(.78)$ & & & & & - & $.39 * *$ \\
\hline 7.PPVT III & $100.7(18.8)$ & & & & & & - \\
\hline
\end{tabular}

Nota. Entre paréntesis rectos [ ] se ofrecen los valores de la correlación parcial controlando según edad en meses y PPVT-III. PPVT III $=$ Test de Vocabulario en Imágenes Peabody, Tercera Edición.

$\dagger p<.10 ; * p<.05 ; * * p<.01$.

Posteriormente, se realizó análisis de correlación parcial para examinar si los valores se deben a la mediación de un constructo latente (e.g.: debido a una habilidad mayor en el dominio del lenguaje). Se espera que si ese constructo latente está influenciado por el lenguaje, los valores se reduzcan considerablemente. Cuando se controlan las puntuaciones según lenguaje receptivo, se destacan dos elementos (a) los valores de correlación de Recuperación del Animal se reducen considerablemente, sugiriendo que el desarrollo de la memoria prospectiva está más ligado al del lenguaje que a la PE y; (b) se conserva la significación entre las tareas destinadas a la evaluación de la PE (tarea de viaje y de dibujo).

Otro aspecto resaltable es la correlación entre la tarea de contenido inesperado y Torre de Hanói. Nuestros datos no se alinean con los de Carlson et al. (2004) sobre el rol del funcionamiento ejecutivo en el desempeño en las tareas de teoría de la mente. En dicho estudio, la capacidad para la teoría de la mente fue predicha por el control inhibitorio y no por medidas de planificación (i.e.: Torre de Hanoi). En nuestro caso, la relación se mantiene significativa. En cambio, sí se confirma la baja relación entre Postergación de la gratificación y la teoría de la mente, reportada por Carlson y Moses (2001). Por otro lado, ¿cómo interpretar que en la correlación parcial la mayoría de las asociaciones pierdan los altos valores que presentaban en las correlaciones de orden cero? Un objetivo de este estudio era determinar si la teoría de la mente y PE tienen un patrón de desarrollo conjunto dado por su pertenencia a un mismo constructo mayor, que las contiene (i.e.: la auto-proyección). Pocos de nuestros datos pueden interpretarse en esa dirección. Una vez desestimada la contribución del lenguaje, las tareas destinadas a evaluar PE no se asocian con las tareas que evaluaban los procesos orientados a futuro o teoría de la mente. Una posible explicación es que estas demanden más funcionamiento ejecutivo: los valores de las correlaciones parciales entre Torre de Hanói con premio diferido y contenido inesperado se mantienen elevados. Esto apunta a que los principales elementos para desempeñar dichas tareas exitosamente no recaen en un mismo constructo, sino - presumiblemente-, sean requeridos otros procesos como el control inhibitorio o la memoria de trabajo. Sin embargo, la PE parece ser requerida, en parte, para la postergación de la gratificación, ya que mantiene valores tendientes a la significación con la tarea de dibujo, luego de ser controlado según lenguaje receptivo.

\section{Discusión General}

Este trabajo tenía 3 objetivos (a) determinar la equivalencia entre diversos tipos de experimentos para evaluar la PE, (b) determinar la coherencia evolutiva en la emergencia de estas competencias en la etapa pre-escolar y estrechamente relacionado con esto (c) determinar si los 
modelos de conceptualización de la PE en constructos más amplios reúnen evidencia empírica en la etapa pre-escolar. Primeramente, se pudo comprobar que las diversas medidas destinadas a evaluar la PE, mantienen valores correlación significativos. Quienes logran desempeñarse bien en la tarea de viaje o en la tarea de los cuartos, también lo hacen en la de dibujo, lo que sugiere que están evaluando la capacidad pretendida, a pesar de que se ha sugerido que la falta de un componente espacial en los experimentos para evaluar la PE afectaría el desempeño (Russell et al., 2010). Esta afirmación así como el reclamo por nuevos experimentos no se ve justificada en función de nuestros datos, debido a la consistente correlación entre las tareas de los cuartos, de dibujo y de viaje, aún cuando se controló según habilidades cognitivas generales. Siendo así, se propone que para la evaluación de la PE en estudios futuros, se utilice una batería que incluya varios experimentos y luego se compute la medida compuesta como variable.

En segundo lugar, se ha mostrado que existe una coherencia comportamental en la aparición ontogenética de la PE, de los procesos orientados a futuro y los de auto-proyección -específicamente en este estudio uno de sus aspectos: la teoría de la mente-. Su emergencia conjunta en los años preescolares podría verse ayudada por la maduración de la capacidad de descentramiento. Ambos estudios vienen a señalar que la prospección es un elemento asociado al desempeño en las tareas de postergación de la gratificación. Sin embargo, queda por explorar el rol mediador que puede tener el PE en el desarrollo de la inhibición: ¿una mayor PE contribuye a una mayor inhibición en la edad preescolar? Se ha establecido cierta relación entre los procesos de percepción temporal, descuento temporal y la impulsividad, tanto en adultos (Wittmann \& Paulus, 2008) como en población infantil, - especialmente su rol en el déficit de atención con hiperactividad (Toplak, Dockstader, \& Tannock, 2006). Así, se vuelve de interés futuro explorar la relación directa entre inhibición, impulsividad, percepción del tiempo y PE, en tanto este estudio muestra que la PE contribuye parcialmente para el éxito en las tareas de postergación de la gratificación.

Por último, no es posible afirmar con nuestros datos la existencia de un constructo latente que explique conjuntamente el rendimiento en la PE, la planificación, la postergación de la gratificación o la teoría de la mente. La PE aparece como un proceso que se desarrolla de forma autónoma a estos: nuestro estudio no reúne evidencia para incluir la PE dentro de un constructo latente ya sea dentro de los procesos de orientación futura ni dentro del de autoproyección. Por otro lado puede sugerirse que el desarrollo de la PE está más ligado al desarrollo lingüístico que a la habilidad general de razonamiento, debido a la diferente reducción de las correlaciones cuando se parcializa según el IMM (Estudio 1) o el lenguaje receptivo (Estudio 2). Sin embargo, esta afirmación debe ser interpretada con cautela debido a que los datos provienen de muestras independientes, por lo que nuevos estudios deben realizarse para avanzar sobre este punto.
Una mención aparte debe ser realizada respecto a las diferencias de género en el desempeño en la tarea de premio diferido. Los efectos encontrados replican hallazgos previos (Silverman, 2003, para una revisión) que muestran que las niñas aventajan a los niños en esta capacidad, incluso en etapas tan tempranas como la pre-escolar. Para este estudio las diferencias de sexo en PE fueron no significativas, lo cual añade evidencia de que la PE y postergación de la gratificación tienen un curso autónomo en el desarrollo pre-escolar.

En suma, este estudio brinda apoyo comportamental respecto a la autonomía en el desarrollo de la PE, al menos con respecto a los procesos de orientación futura y teoría de la mente. Nuestros datos muestran que dichas competencias dejan de estar asociadas cuando son controladas por el lenguaje y tienden a agruparse en las medidas de PE, por un lado y las de funcionamiento ejecutivo y Teoría de la Mente, por otro. Estos datos se alinean con otros trabajos recientes en el campo del desarrollo infantil que sugieren que la PE integra un sistema para la cognición episódica (e.g.: Suddendorf, 2010). Por otro lado, las diferencias inter-individuales encontradas abren la puerta a nuevos estudios que intenten explicar qué variables evolutivas son las que contribuyen a un desarrollo temprano de la capacidad. Investigaciones futuras que intenten realizar correlaciones predictivas para conocer si la adquisición temprana de la PE tiene efectos sobre el desempeño en las habilidades sociales, la perspectiva temporal o las competencias cognitivas en momentos posteriores del desarrollo serán de gran relevancia.

\section{Referencias}

Atance, C., \& Jackson, L. (2009). The development and coherence of future-oriented behaviors during the preschool years. Journal of Experimental Child Psychology, 102, 379-391. doi:10.1016/j.jecp.2009.01.001

Atance, C., \& Meltzoff, A. (2005). My future self: Young children's ability to anticipate and explain future states. Cognitive Development, 20, 341-361. doi:10.1016/j.cogdev.2005.05.001

Atance, C., \& O'Neill, D. (2001). Episodic future thinking. Trends in Cognitive Science, 5, 533-539. doi:10.1016/S13646613(00)01804-0

Atance, C., \& O'Neill, D. (2005). The emergence of episodic future thinking in humans. Learning and Motivation, 36, 126-144. doi:10.1016/j.lmot.2005.02.003

Bembenutty, H., \& Karabenick, S. (2004). Inherent association between academic delay of gratification, future time perspective and self-regulated learning. Educational Psychology Review, 16, 35-57. doi:10.1023/B:EDPR.0000012344.34008.5c

Bischof, D. (2008, July). Perspective taking, mental time travel, gender constancy and child-parent-relationship in four year olds: How they connect. Paper presented at the International Congress of Psychology, Berlin, Germany.

Buckner, R., \& Carroll, D. (2007). Self-projection and the brain. Trends in Cognitive Sciences, 11, 49-57. doi:10.1016/j. tics.2006.11.004

Burgemeister, B., Hollander, L., \& Lorge, I. (1983). Escala de Madurez Mental de Columbia. Madrid, España: TEA. 
Echeverría, A. V. (2015). La Previsión Episódica en la Etapa Pre-Escolar: Equivalencia entre Medidas y su Relación con los Procesos de Orientación Futura y la Teoría de la Mente.

Carlson, S. M., \& Moses, L. J. (2001). Individual differences in inhibitory control and children's theory of mind. Child Development, 72, 1032-1053. doi:10.1111/1467-8624.00333

Carlson, S. M., Moses, L. J., \& Claxton, L. J. (2004). Individual differences in executive functioning and theory of mind: An investigation of inhibitory control and planning ability. Journal of Experimental Child Psychology, 87, 299-319. doi:10.1016/j.jecp.2004.01.002

Dunn, L. M., Dunn, L. M., \& Arribas, D. (2006). PPVT-III PEABODY. Test de vocabulario en imágenes Peabody (D. Arribas, Trans.). Madrid, España: TEA.

Gopnik, A., \& Astington, J. W. (1988). Children's understanding of representational change and its relation to the understanding of false belief and the appearance-reality distinction. Child Development, 59, 26-37. doi:10.1111/j.1467-8624.1988. tb03192.x

Heith, M. (1997). The development of future thinking as essential for the emergence of skill in planning. In S. L. Friedman \& E. K. Scholnick (Eds.), The developmental psychology of planning: Why, how, and when do we plan? (pp. 25-42). Mahwah, NJ: Erlbaum.

Hudson, J. A., Shapiro, L., \& Sosa, B. (1995). Planning in the real world: Preschool children's scripts and plans for familiar events. Child Development, 66, 984-998. doi:10.1111/j.1467-8624.1995.tb00917.x

Kliegel, M., Martin, M., McDaniel, M. A., \& Einstein, G. O. (2002). Complex prospective memory and executive control of working memory: A process model. Psychologische Beiträge, 44, 303-318.

Landis, J. R., \& Koch, G. G. (1977). The measurement of observer agreement for categorical data. Biometrics, 33, 159-174. doi: $10.2307 / 2529310$

McCormack, T., \& Hoerl, C. (2008). Temporal decentering and the development of temporal concepts. Language Learning, 58(Suppl. 1), 89-113. doi:10.1111/j.1467-9922.2008.00464.x

Mischel, W., Shoda, Y., \& Peake, P. (1988). The nature of adolescents competencies predicted by preschool delay of gratification. Journal of Personality and Social Psychology, 54, 687-696. doi:10.1037/0022-3514.54.4.687

Ródenas, A., Brito, A. G., \& Carranza, J. A. (1991). La referencia temporal de futuro en el lenguaje espontáneo infantil. Anales de Psicología, 7, 225-241.

Rosenbaum, R. S., Stuss, D. T., Levine, B., \& Tulving, E. (2007). Theory of Mind is independent of episodic memory. Science, 318, 1257. doi: $10.1126 /$ science. 1148763

Russell, J., Alexis, D., \& Clayton, N. (2010). Episodic future thinking in 3 to 5-year-old children: The ability to think of what will be needed from a different point of view. Cognition, 114, 56-71. doi:10.1016/j.cognition.2009.08.013

Scholl, B. J., \& Leslie, A. M. (1999). Modularity, development and Theory of Mind. Mind and Language, 14, 131-153. doi:10.1111/1468-0017.00106

Silverman, I. (2003). Gender differences in delay of gratification: A meta-analysis. Sex Roles, 49, 451-463. doi:10.1023/A:1025872421115

Suddendorf, T. (2010). Episodic memory versus episodic foresight: Similarities and differences. Wiley Interdisciplinay Reviews Cognitive Science, 1, 99-107. doi:10.1002/wcs.23

Suddendorf, T., \& Busby, J. (2005). Making decisions with the future in mind: Developmental and comparative identification of mental time travel. Learning \& Motivation, 36, 110-125. doi:10.1016/j.lmot.2005.02.010
Suddendorf, T., \& Corballis, M. (2007). The evolution of foresight: What is mental time travel, and is it unique to humans? Behavioral and Brain Sciences, 30, 299-351. doi:10.1017/ S0140525X07001975

Suddendorf, T., \& Moore, C. (2011). Introduction to the special issue: The development of episodic foresight. Cognitive Development, 26, 295-298. doi:10.1016/j.cogdev.2011.09.001

Szpunar, K. K. (2010). Episodic future thought: An emerging concept. Perspectives on Psychological Science, 5, 142-162. doi:10.1177/1745691610362350

Teglás, E., Girotto, V., Gonzalez, M., \& Bonatti, L. (2007). Intuitions of probabilities shape expectations about the future at 12 months and beyond. Proceedings of the National Academy of Sciences, 104, 19156-19159. doi:10.1073/pnas.0700271104

Toplak, M. E., Dockstader, C., \& Tannock, R. (2006). Temporal processing in ADHD: Findings to date and new methods. Journal of Neuroscience Methods, 151, 15-29. doi:10.1016/j. jneumeth.2005.09.018

Tulving, E. (1984). Précis of elements of episodic memory. Behavioral and Brain Sciences, 7, 223-268. doi:10.1017/ S0140525X0004440X

Wittmann, M., \& Paulus, M. P. (2008). Decision making, impulsivity and time perception. Trends in Cognitive Science. 12, 7-12. doi:10.1016/j.tics.2007.10.004 